\title{
Um problema não trivial de projécteis
}

\author{
(A non trivial projectile problem)
}

\author{
Manuel Fernando Ferreira da Silval e Eduardo Rino Alberto Segre \\ Departamento de Física, Universidade da Beira Interior, Covilhã, Portugal \\ Recebido em 18/05/04; Aceito em 19/07/04
}

Resolve-se de uma forma original um problema de projécteis pouco conhecido e de alguma dificuldade.

Palavras-chave: Cinemática, movimento de projécteis.

An uncommon projectile motion problem of unexpected difficulty is solved in an original way.

Keywords: Kinematics, projectile motion.

\section{Introdução}

O estudo do movimento de um projéctil num campo gravítico constante desprezando a resistência do ar é uma das aplicações mais elementares da cinemática de uma partícula, e constitui um assunto obrigatório em todos os livros de texto de Física Geral a nível intermédio. Este estudo é importante porque estende para duas dimensões o problema da queda livre unidimensional, e permite que os estudantes se familiarizem com a "separação" de dois movimentos efectuados em direcções perpendiculares.

$\mathrm{O}$ facto das equações de movimento serem bastante simples leva com frequência a pensar que todos os problemas de projécteis são de certa forma triviais; há, no entanto, algumas excepções. Neste trabalho pretende-se mostrar uma dessas excepções.

\section{Formulação do problema}

Consideremos um projéctil que é lançado desde o topo de um hemisfério de raio $R$. Sobre o projéctil actua um campo gravítico vertical constante $\vec{g}$ (ver Fig. 1). Deseja-se determinar a mínima velocidade de lançamento $v_{0}$ que o projéctil deverá ter de modo a conseguir abandonar o hemisfério sem colidir com o mesmo.
Este problema é muito pouco conhecido, e um dos aspectos que o torna especial é o facto da abordagem tradicional (puramente geométrica) ser extremamente complicada (no apêndice é esboçada essa solução geométrica). Aqui o problema será resolvido de uma forma alternativa, bem mais física; a solução que apresentamos apoia-se na solução prévia de outros dois problemas, algo mais conhecidos, o que torna esta abordagem bastante apelativa e, do ponto de vista pedagógico, muito instrutiva.

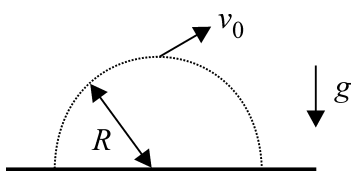

Figura 1 - Projéctil lançado do topo de um hemisfério.

\footnotetext{
${ }^{1}$ Enviar correspondência para Manuel Fernando Ferreira da Silva. E-mail: ferreira@dfisica.ubi.pt.
} 
medido ao longo da rampa.

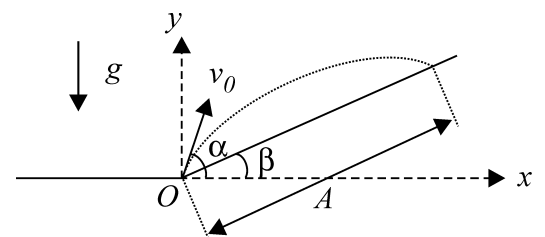

Figura 2 - Projéctil lançado sobre uma rampa.

Este é um problema clássico [1-6]. Escolhendo $t=0$ como instante de lançamento, e o referencial $O x y$ da Fig. 2, o vector posição do projéctil será, em função do tempo,

$$
\vec{r}(t)=\left(v_{0} \cos \alpha\right) t \hat{x}+\left[\left(v_{0} \sin \alpha\right) t-\frac{1}{2} g t^{2}\right] \hat{y},
$$

de modo que a equação da trajectória será a parábola

$$
y=(\tan \alpha) x-\frac{g}{2 v_{0}^{2} \cos ^{2} \alpha} x^{2} .
$$

A intersecção desta parábola com a recta $y=$ $(\tan \beta) x$ que representa a rampa resulta num ponto cuja abcissa satisfaz a equação

$$
\frac{g}{2 v_{0}^{2} \cos ^{2} \alpha} x^{2}=(\tan \alpha-\tan \beta) x,
$$

da qual resulta, para além da solução trivial $x=0$, a solução $x_{\mathrm{i}}$ associada ao ponto de impacto:

$$
\begin{aligned}
x_{\mathrm{i}} & =\frac{2 v_{0}^{2} \cos ^{2} \alpha(\tan \alpha-\tan \beta)}{g} \\
& =\frac{v_{0}^{2}}{g}\left[2 \sin \alpha \cos \alpha-2 \cos ^{2} \alpha \frac{\sin \beta}{\cos \beta}\right] \\
& =\frac{v_{0}^{2}}{g} \frac{\sin (2 \alpha) \cos \beta-\sin \beta[1+\cos (2 \alpha)]}{\cos \beta} \\
& =\frac{v_{0}^{2}}{g} \frac{\sin (2 \alpha-\beta)-\sin \beta}{\cos \beta} .
\end{aligned}
$$

Logo, o alcance do projéctil ao longo da rampa será

$$
A=\frac{x_{\mathrm{i}}}{\cos \beta}=\frac{v_{0}^{2}}{g} \frac{\sin (2 \alpha-\beta)-\sin \beta}{\cos ^{2} \beta} .
$$

Para uma velocidade $v_{0}$ fixa, o máximo alcance $A_{\text {máx }}$ é atingido quando $\sin (2 \alpha-\beta)=1$, ou seja, quando $2 \alpha-\beta=\frac{\pi}{2}$; assim $A_{\text {máx }}$ é atingido quando:

$$
\alpha=\frac{\pi}{4}+\frac{\beta}{2}
$$

e o seu valor será

$$
A_{\text {máx }}=\frac{v_{0}^{2}}{g} \frac{1-\sin \beta}{1-\sin ^{2} \beta}=\frac{v_{0}^{2}}{g} \frac{1}{\sin \beta+1} .
$$

O caso especial de lançamento sobre uma superfície horizontal $(\beta=0)$ proporciona os resultados bem conhecidos

$$
A_{\text {máx }}=\frac{v_{0}^{2}}{g}
$$

quando:

$$
\alpha=\frac{\pi}{4} \quad(\beta=0) .
$$

Naturalmente, todos os resultados anteriores permitem também valores negativos de $\beta$, o que fisicamente equivale a fazer o lançamento desde o topo da rampa e não a partir da base, que é a forma em que o problema é apresentado em alguns livros [7-9].

\section{Problema auxiliar 2: Disparando por cima de um obstáculo}

Deseja-se que um projéctil passe por cima de um obstáculo de altura $h$ localizado a uma distância horizontal $d$ do ponto de lançamento (ver Fig. 3). Qual é a mínima velocidade de lançamento $v_{0}$ que torna isso possível?

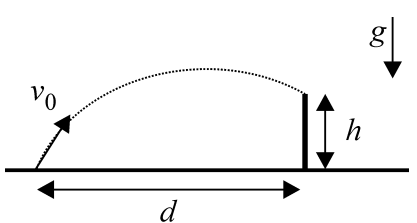

Figura 3 - Projéctil lançado por cima de um obstáculo.

Embora menos conhecido que o problema anterior, este problema pode também ser encontrado na literatura [10]. Para o resolver, em vez de atacá-lo directamente aproveitamos os resultados da secção anterior. Assim, traçamos uma semi-recta com origem no ponto de lançamento que passe pelo ponto mais alto do obstáculo. Esta semi-recta será o equivalente da rampa do problema auxiliar 1, e a sua inclinação será $\beta=\arctan (h / d)$. Vejamos qual a condição para que o projéctil passe pelo ponto mais alto do obstáculo.

Numa tal situação, o alcance $A$ ao longo da semirecta é fixado, o ângulo de lançamento ideal (de forma a minimizar o valor de $v_{0}$ ) será, de acordo com (6),

$$
\alpha=\frac{\pi}{4}+\frac{1}{2} \arctan \left(\frac{h}{d}\right),
$$


e o valor mínimo de $v_{0}$ obtém-se a partir de (7):

$$
\begin{aligned}
v_{0, \text { mín }} & =\sqrt{g A(\sin \beta+1)} \\
& =\sqrt{g(A \sin \beta+A)} \\
& =\sqrt{g\left(h+\sqrt{h^{2}+d^{2}}\right)} .
\end{aligned}
$$

Assim, para que o projéctil ultrapasse o obstáculo bastará escolher

$$
v_{0}>\sqrt{g\left(h+\sqrt{h^{2}+d^{2}}\right)},
$$

com o ângulo de lançamento (9).

\section{Resolução do problema inicial: Dis- parando do topo de um hemisfério}

Voltemos agora ao problema enunciado na secção 2. Tendo em conta a resolução da secção 4, podemos agora reformular este problema imaginando o hemisfério como um conjunto (infinito) de obstáculos cujos pontos mais altos se encontram a alturas cada vez mais pequenas (ver Fig. 4).

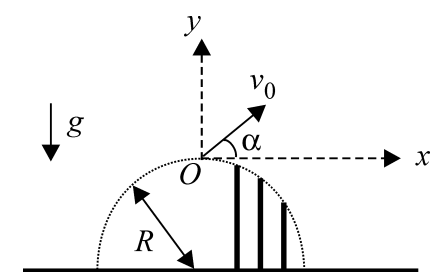

Figura 4 - Resolvendo o problema proposto.

Escolhendo o referencial $O x y$ representado, o ponto mais alto do obstáculo colocado na posição $x=$ $d$ terá uma coordenada $h=y, \operatorname{com} x^{2}+(y+R)^{2}=R^{2}$. Assim,

$$
\begin{array}{r}
d^{2}=R^{2}-(h+R)^{2}=-h^{2}-2 R h \\
\Longrightarrow \quad h^{2}+d^{2}=-2 R h .
\end{array}
$$

Convém notar que os valores $h$ dos obstáculos são, neste caso, negativos.

Para cada obstáculo, a velocidade mínima $v_{0}$ requerida virá dada por (11); logo, para cada $h$ no intervalo $[-R, 0]$, teremos

$$
v_{0}^{2}>g(h+\sqrt{-2 R h}) .
$$

Resta agora determinar o valor de $h$ para o qual a função

$$
f(h)=h+\sqrt{-2 R h}=\frac{R}{2}-\left(\sqrt{\frac{R}{2}}-\sqrt{-h}\right)^{2}
$$

atinge o valor máximo, e usar depois (13) para obter o valor mínimo de $v_{0}$. Obviamente $f(h)$ é máximo quando

$$
\begin{array}{r}
\sqrt{\frac{R}{2}}=\sqrt{-h} \quad \Longrightarrow \quad \frac{R}{2}=-h \\
\Longrightarrow \quad h=-\frac{1}{2} R,
\end{array}
$$

valor para o qual corresponde

$$
f\left(-\frac{R}{2}\right)=\frac{R}{2},
$$

de modo que (13) dá

$$
v_{0}^{2}>\frac{g R}{2} .
$$

Usando (12) obtém-se o valor correspondente de $d$ :

$$
d=\sqrt{R^{2}-\frac{R^{2}}{4}}=\frac{\sqrt{3}}{2} R .
$$

$\mathrm{O}$ ângulo de lançamento associado a esta velocidade mínima é, usando (9), (15) e (18),

$$
\begin{aligned}
\alpha= & \frac{\pi}{4}+\frac{1}{2} \arctan \left(\frac{-R / 2}{\sqrt{3} R / 2}\right) \\
& =\frac{\pi}{4}+\frac{1}{2}\left(-\frac{\pi}{6}\right)=\frac{\pi}{6} .
\end{aligned}
$$

Concluindo, para que o projéctil abandone o hemisfério com velocidade mínima deverá ser lançado com um ângulo de $30^{\circ}$ e com uma velocidade $v_{0}$ tal que

$$
v_{0}>\sqrt{\frac{g R}{2}} .
$$

\section{Conclusões}

Foi resolvido um problema de projécteis muito pouco conhecido, e que está longe de ser trivial; o processo de resolução envolveu o estudo de outros dois problemas de projécteis ligeiramente mais simples. Desta forma, um problema complicado acabou por tornar-se fácil. No apêndice mostram-se as grandes dificuldades que apresenta o ataque directo do problema proposto.

Convém salientar que tanto o problema proposto como os dois problemas auxiliares foram resolvidos sem recorrer ao cálculo diferencial; assim, qualquer destes problemas pode, em princípio, ser explicado a alunos que ainda não conheçam o conceito de derivada. 
Já a abordagem geométrica ensaiada no apêndice requer necessariamente não só o conceito de derivada como também o teorema da função implícita.

Espera-se que este exemplo tenha mostrado que os problemas de projécteis podem ser algo mais do que simples "rotina"; deseja-se também que ele tenha ilustrado como três problemas bastante diferentes podem ser estreitamente relacionados, criando assim uma visão de conjunto que pensamos ser muito pedagógica.

\section{Referências}

[1] M.R. Spiegel, Theory and Problems of Theoretical Mechanics (problem 3.21, McGraw-Hill, New York, 1967).

[2] J. Norwood, Jr., Intermediate Classical Mechanics (problem 2-2-5, Prentice-Hall, Englewood Cliffs, New Jersey, 1979).

[3] M. Alonso and E.J. Finn, Physics (problem 4.10, Addison-Wesley, Reading, Massachusetts, 1992).

[4] R. Resnick, D. Halliday and K.S. Krane, Physics (problem 44, Wiley, New York, 1992), 4th ed., chapter 4.

[5] W.G. Rees, Physics by Example: 200 Problems and Solutions (problem 15, Cambridge University Press, Cambridge, 1994).

[6] J.B. Marion and S.T. Thornton, Classical Dynamics of Particles and Systems (problem 2-14, Saunders College Pub., Fort Worth, Philadelphia, 1995), 4th ed.

[7] Referência 1, problem 3.64.

[8] D. Kleppner and R.J. Kolenkow, An Introduction to Mechanics (problem 1.21, McGraw-Hill, Boston, 1973).

[9] ] V.D. Barger and M. G. Olsson, Classical Mechanics - A Modern Perspective (problem 1-4, McGraw-Hill, New York, 1995), 2nd ed.

[10] B.B. Bukhovtsev, V.D. Krivtchenkov, G.Ya. Miakishev e I.M. Saraeva, Problemas Selecionados de Física Elementar (problema 42, Editora Mir, Moscovo, 1977).

\section{Apêndice}

Descrevemos aqui, muito resumidamente, como pode ser resolvido o problema proposto através de uma análise puramente geométrica. Começamos por escrever a equação da trajectória parabólica descrita pelo projéctil, usando o referencial $O x y$ da Fig. 4:

$$
y=(\tan \alpha) x-\frac{g}{2 v_{0}^{2} \cos ^{2} \alpha} x^{2} .
$$

Esta trajectória deve ser interceptada com a circunferência

$$
x^{2}+(y+R)^{2}=R^{2} .
$$

Introduzindo (21) em (22) e desenvolvendo, chegase à equação

$$
\begin{gathered}
a x^{3}-b x^{2}-c x+d=0, \\
a=\left(\frac{g}{2 v_{0}^{2} \cos ^{2} \alpha}\right)^{2}, \\
b=\frac{g \tan \alpha}{v_{0}^{2} \cos ^{2} \alpha}, \\
c=\frac{g R-v_{0}^{2}}{v_{0}^{2} \cos ^{2} \alpha}, \\
d=2 R \tan \alpha .
\end{gathered}
$$

A Eq. (23) descreve os pontos de intersecção entre $(21)$ e (22) diferentes do ponto de lançamento $(x=0)$. A Eq. (23) poderá ter três raízes reais diferentes (Fig. $5 a$ ), duas raízes reais iguais e outra real diferente (Fig. $5 b$ ), e uma raiz real e duas complexas conjugadas (Fig. $5 c$ ). Estamos interessados na situação limite mostrada na Fig. 5b. Sejam $x=-x_{1}$ e $x=x_{2}$ as duas soluções representadas nessa figura.

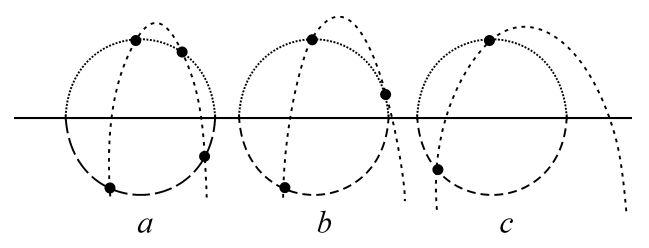

Figura 5 - Resolução geométrica do problema.

A Eq. (23) deverá então poder ser escrita na forma

$$
a\left(x+x_{1}\right)\left(x-x_{2}\right)^{2}=0 .
$$

Igualando (23) e (28) obtêm-se as seguintes equações:

$$
\begin{gathered}
x_{1}-2 x_{2}=-\frac{b}{a}, \\
x_{2}^{2}-2 x_{1} x_{2}=-\frac{c}{a}, \\
x_{1} x_{2}^{2}=\frac{d}{a} .
\end{gathered}
$$

Combinando (29) e (30) é possível retirar $x_{1}$ e $x_{2}$ :

$$
\begin{array}{r}
x_{1}=-\left(\frac{b}{3 a}\right)+2 \sqrt{\left(\frac{b}{3 a}\right)^{2}+\left(\frac{c}{3 a}\right)} ; \\
x_{2}=\left(\frac{b}{3 a}\right)+\sqrt{\left(\frac{b}{3 a}\right)^{2}+\left(\frac{c}{3 a}\right)},
\end{array}
$$


e inserindo as expressões (32) em (31) obtém-se a condição

$$
\begin{gathered}
4\left[\left(\frac{b}{3 a}\right)^{2}+\left(\frac{c}{3 a}\right)\right]^{3}= \\
{\left[\left(\frac{d}{a}\right)-3\left(\frac{b}{3 a}\right)\left(\frac{c}{3 a}\right)-2\left(\frac{b}{3 a}\right)^{3}\right]^{2} .}
\end{gathered}
$$

Quando as expressões (24)-(27) são substituídas em (33) resulta

$$
\begin{gathered}
\frac{4}{3}\left(z-1+\frac{4}{3} \sin ^{2} \alpha\right)^{3}= \\
\sin ^{2} \alpha\left(z+2-\frac{16}{9} \sin ^{2} \alpha\right)^{2},
\end{gathered}
$$

onde se definiu

$$
z=\frac{g R}{v_{0}^{2}} .
$$

A relação (34) define implicitamente $z$ em função de $\alpha$, e pode ser posta na forma

$$
\begin{gathered}
4(z-1)^{3}+\left(13 z^{2}-44 z+4\right) \sin ^{2} \alpha+32 z \\
\sin ^{4} \alpha=0 .
\end{gathered}
$$

O nosso objectivo é saber em que condições $z$ atinge o seu valor máximo (valor mínimo de $v_{0}$ ). Derivando implicitamente (36) com respeito a $\alpha$ e impondo a condição $\frac{\mathrm{d} z}{\mathrm{~d} \alpha}=0$ resulta

$$
\sin ^{2} \alpha=-\frac{13 z^{2}-44 z+4}{64 z},
$$

que inserida novamente em (36) permite obter a equação

$$
\begin{array}{r}
343 z^{4}-392 z^{3}-504 z^{2}-160 z-16=0 \\
\Longrightarrow \quad(z-2)(7 z+2)^{3}=0 .
\end{array}
$$

A Eq. (38) tem apenas duas raízes: $z=2 \mathrm{e}$ $z=-2 / 7$. Só a primeira é admissível, devido a (35), o que determina o valor mínimo de $v_{0}$ :

$$
2=\frac{g R}{v_{0, \text { mín }}^{2}} \quad \Longrightarrow \quad v_{0, \text { mín }}=\sqrt{\frac{g R}{2}} .
$$

Substituindo o valor obtido $z=2$ em (37) resulta

$$
\sin ^{2} \alpha=\frac{1}{4} \quad \Longrightarrow \quad \alpha=\frac{\pi}{6} .
$$

